\title{
B-SPLINE COLLOCATION METHODS FOR NUMERICAL SOLUTIONS OF THE BURGERS' EQUATION
}

\author{
İDRİS DAĞ, DURSUN IRK, AND ALİ ŞAHİN
}

Received 25 August 2004 and in revised form 22 December 2004

Both time- and space-splitted Burgers' equations are solved numerically. Cubic B-spline collocation method is applied to the time-splitted Burgers' equation. Quadratic B-spline collocation method is used to get numerical solution of the space-splitted Burgers' equation. The results of both schemes are compared for some test problems.

\section{Introduction}

The Burgers' equation first appeared in the paper by Bateman [3], who mentioned two of the essentially steady solutions. Due to extensive works of Burgers [4] involving the Burgers' equation especially as a mathematical model for the turbulence, it is known as Burgers' equation. The equation is used as a model in fields as wide as heat conduction [5], gas dynamics [13], shock waves [4], longitudinal elastic waves in an isotropic solid [15], number theory [18], continues stochastic processes [5], and so forth. Hopf [8] and Cole [5] solved the Burgers' equation analytically and independently for arbitrary initial conditions. In many cases, these solutions involve infinite series which may converge very slowly for small values of viscosity coefficients $v$, which correspond to steep wave fronts in the propagation of the dynamic wave forms. Burgers' equation shows a similar features with Navier-Stokes equation due to the form of the nonlinear convection term and the occurrence of the viscosity term. Before concentrating on the numerical solution of the Navier-Stokes equation, it seems reasonable to first study a simple model of the Burgers' equation. Therefore, the Burgers' equation has been used as a model equation to test the numerical methods in terms of accuracy and stability for the Navier-Stokes equation. Many authors have used a variety of numerical techniques for getting the numerical solution of the Burgers' equation. Numerical difficulties have been come across in the numerical solution of the Burgers' equation with a very small viscosity. Various numerical techniques accompanied with spline functions have been set up for computing the solutions of the Burgers' equation. Rubin and Graves have used the cubic spline function technique and quasilinearisation for the numerical solutions of the Burgers' equation in one space variable at low Reynolds numbers [16]. A cubic spline collocation procedure has been developed for the numerical solution of the Burgers' equation [17]. A combination 
of the time-splitted scheme and cubic spline functions was used to set up implicit finite difference schemes for obtaining the numerical solution of the Burgers' equation in the papers $[9,10,11,14]$. The equation is solved numerically by the collocation method with cubic B-spline interpolation functions over uniform elements by Ali et al. [2]. A finite element solution of the Burgers' equation based on Galerkin method using B-splines as both element shape and test functions is developed in the studies [1, 6, 7]. Least-squares formulation using quadratic B-splines as trial function is given over the finite intervals by Kutluay et al. [12]. Numerical solutions of the partial differential equations have been found by splitting the equation both to make the numerical method applicable and to increase the accuracy of the method. We have written two algorithms for the splitted Burgers' equation. First, Burgers' equation is splitted in time and then cubic B-spline collocation method is applied. To be able to use the quadratic B-splines as trial functions in the collocation method, setting $V=-U_{x}$ in the Burgers' equation gives a first-order coupled system. This system of equations involving the first-order derivatives can be computed by employing the quadratic B-spline collocation method. Numerical results for some known initial and boundary conditions are illustrated for both methods.

Briefly, outline is as follows. In Section 2, numerical methods are described. Numerical experiments are carried out for two test problems and results of those methods are compared with each other and with theoretical results in Section 3.

\section{B-spline collocation methods}

The form of one-dimensional Burgers' equation is

$$
U_{t}+U U_{x}-\nu U_{x x}=0
$$

where $v>0$ is the coefficient of the kinematic viscosity and subscripts $x$ and $t$ denote differentiation. Initial and boundary conditions are chosen as follows:

$$
\begin{gathered}
U(x, 0)=f(x), \quad a \leq x \leq b, \\
U(a, t)=\alpha_{1}, \quad U(b, t)=\alpha_{2}, \quad t \in[0, T] .
\end{gathered}
$$

We consider a mesh $a=x_{0}<x_{1} \cdots<x_{N}=b$ as a uniform partition of the solution domain $a \leq x \leq b$ by the knots $x_{m}$ and $h=x_{m}-x_{m-1}, m=1, \ldots, N$, throughout paper.

2.1. Quadratic B-spline collocation method (QBCM). A direct application of the quadratic B-spline collocation method requires the first-order derivatives in the equation to obtain smooth solutions. To start with formulation of the quadratic B-spline collocation method for the Burgers' equation, the space splitting is done with setting $V=-U_{x}$. So that Burgers' equation turns into the system of equations which involves the first-order derivatives. In the system of equations, the unknown functions $U, V$ and their space derivatives $U_{x}, V_{x}$ are discretized by the quadratic B-splines. 
Let $Q_{m}(x), m=-1, \ldots, N$,

$$
Q_{m}(x)=\frac{1}{h^{2}} \begin{cases}\left(x_{m+2}-x\right)^{2}-3\left(x_{m+1}-x\right)^{2}+3\left(x_{m}-x\right)^{2}, & {\left[x_{m-1}, x_{m}\right],} \\ \left(x_{m+2}-x\right)^{2}-3\left(x_{m+1}-x\right)^{2}, & {\left[x_{m}, x_{m+1}\right],} \\ \left(x_{m+2}-x\right)^{2}, & {\left[x_{m+1}, x_{m+2}\right],} \\ 0, & \text { otherwise }\end{cases}
$$

be quadratic B-splines with the knots $x_{m}, m=0, \ldots, N$. A basis is formed with quadratic B-splines $Q_{m}(x)$ over the domain region $a \leq x \leq b$.

The first-order space splitting of the Burgers' equation, with $V(x, t)=-U_{x}(x, t)$, gives a coupled system for $U$ and $V$ :

$$
\begin{gathered}
U_{t}-U V+\nu V_{x}=0, \\
V+U_{x}=0
\end{gathered}
$$

and boundary and initial conditions are

$$
\begin{gathered}
U(a, t)=\alpha_{1}, \quad U(b, t)=\alpha_{2}, \quad V(a, t)=V(b, t)=0, \quad t \in[0, T], \\
U(x, 0)=f(x), \quad V(x, 0)=-f^{\prime}(x), \quad a \leq x \leq b .
\end{gathered}
$$

The collocation method is applied to find approximate solutions of the system (2.5). Collocation approximant can be expressed for $U(x, t)$ and $V(x, t)$ in terms of element parameters $\delta_{m}$ and $\sigma_{m}$, respectively, and quadratic B-splines $Q_{m}(x), m=-1, \ldots, N$ :

$$
U_{N}(x, t)=\sum_{m=-1}^{N} \delta_{m}(t) Q_{m}(x), \quad V_{N}(x, t)=\sum_{m=-1}^{N} \sigma_{m}(t) Q_{m}(x) .
$$

Element parameters $\delta_{m}$ and $\sigma_{m}$ are found by requiring that $U_{N}$ and $V_{N}$ satisfy the system of (2.5) at knots $x_{m}, m=0, \ldots, N$. The nodal variables $U_{m}, V_{m}$ and their derivatives $U_{m}^{\prime}, V_{m}^{\prime}$ have the following representation obtained with substitution of the knots in (2.7) and their first derivative in terms of elements parameters:

$$
\begin{gathered}
U_{m}=U\left(x_{m}\right)=\delta_{m-1}+\delta_{m}, \\
h U_{m}^{\prime}=h U^{\prime}\left(x_{m}\right)=2\left(\delta_{m}-\delta_{m-1}\right), \\
V_{m}=V\left(x_{m}\right)=\sigma_{m-1}+\sigma_{m}, \\
h V_{m}^{\prime}=h V^{\prime}\left(x_{m}\right)=2\left(\sigma_{m}-\sigma_{m-1}\right) .
\end{gathered}
$$

Substituting the collocation approximants (2.7)-(2.8) in the system (2.5) and its evaluation at the knots give a nonlinear system of equations:

$$
\begin{gathered}
h\left(\stackrel{\circ}{\delta}_{m-1}+\stackrel{\circ}{\delta}_{m}\right)-h z_{m}\left(\sigma_{m-1}+\sigma_{m}\right)+2 \nu\left(-\sigma_{m-1}+\sigma_{m}\right)=0, \\
h\left(\sigma_{m-1}+\sigma_{m}\right)+2\left(\delta_{m}-\delta_{m-1}\right)=0,
\end{gathered}
$$

where $\circ$ denotes differentiation with respect to time and $z_{m}=\delta_{m-1}+\delta_{m}$ is known as nonlinear term. 
Time discretization of parameters $\delta_{m}$ and $\sigma_{m}$ of the obtained system (2.9) is done with interpolation between two successive time levels $n$ and $n+1$. So, replacing the following time centering on $t=(n+1 / 2) \Delta t$ and its time derivative with a Crank-Nicholson approach into (2.9),

$$
\begin{aligned}
& \delta_{m}=\frac{\delta_{m}^{n+1}+\delta_{m}^{n}}{2}, \quad \stackrel{\circ}{\delta}_{m}=\frac{\delta_{m}^{n+1}-\delta_{m}^{n}}{\Delta t}, \\
& \sigma_{m}=\frac{\sigma_{m}^{n+1}+\sigma_{m}^{n}}{2}, \quad \stackrel{\circ}{\sigma}_{m}=\frac{\sigma_{m}^{n+1}-\sigma_{m}^{n}}{\Delta t},
\end{aligned}
$$

leads to nonlinear system of $2 N+2$ algebraic equations in the $2 N+4$ unknowns:

$$
\begin{gathered}
2 h \delta_{m-1}^{n+1}-\beta_{m 1} \sigma_{m-1}^{n+1}+2 h \delta_{m}^{n+1}+\beta_{m 2} \sigma_{m}^{n+1}=2 h \delta_{m-1}^{n}+\beta_{m 1} \sigma_{m-1}^{n}+2 h \delta_{m}^{n}-\beta_{m 2} \sigma_{m}^{n}, \\
-2 \delta_{m-1}^{n+1}+h \sigma_{m-1}^{n+1}+2 \delta_{m}^{n+1}+h \sigma_{m}^{n+1}=2 \delta_{m-1}^{n}-h \sigma_{m-1}^{n}-2 \delta_{m}^{n}-h \sigma_{m}^{n+1}, \quad m=0, \ldots, N,
\end{gathered}
$$

where

$$
\beta_{m 1}=z_{m} h \Delta t+2 v \Delta t, \quad \beta_{m 2}=-z_{m} h \Delta t+2 v \Delta t, \quad z_{m}=\delta_{m-1}+\delta_{m}
$$

The boundary conditions $U_{0}=\delta_{-1}+\delta_{0}$ and $V_{N}=\sigma_{N-1}+\sigma_{N}$ are imposed to eliminate parameters $\delta_{-1}$ and $\sigma_{N}$ from the system (2.11) to have an equal solvable $(2 N+2) \times(2 N+$ 2) penta-diagonal matrix system.

To iterate the system (2.11), we need to compute the initial parameters $\delta_{m}^{0}, \sigma_{m}^{0}$. To do so, the following requirements of initial and boundary conditions at time $t=0$ are used:

$$
\begin{gathered}
\left(U_{N}\right)_{x}(a, 0)=0, \quad\left(V_{N}\right)_{x}(a, 0)=0, \\
U_{N}(x, 0)=U\left(x_{m}, 0\right), \quad V_{N}(x, 0)=V\left(x_{m}, 0\right), \quad m=0, \ldots, N,
\end{gathered}
$$

these requirements gives the following determination of the unknown element parameters:

$$
\begin{gathered}
\delta_{-1}^{0}=\frac{U(a, 0)}{2}, \quad \delta_{0}^{0}=\frac{U(a, 0)}{2}, \quad \delta_{m}^{0}=U\left(x_{m}, 0\right)-\delta_{m-1}^{0}, \\
\sigma_{-1}^{0}=\frac{V(a, 0)}{2}, \quad \sigma_{0}^{0}=\frac{V(a, 0)}{2}, \quad \sigma_{m}^{0}=V\left(x_{m}, 0\right)-\sigma_{m-1}^{0}, \quad m=0, \ldots, N .
\end{gathered}
$$

Once element parameters are determined, time evolutions of the $\delta_{m}^{n}, \sigma_{m}^{n}$ are found from the system. Any nodal value and its derivatives can be recovered from (2.8) during the running of the program. To cope with the nonlinearity of the system (2.11), solutions can be made better by using the following two or three iterations at each time $n+1$ for the parameters $\delta_{m}^{n+1}, \sigma_{m}^{n+1}$ :

$$
\left(\boldsymbol{\delta}^{*}\right)^{n+1}=\boldsymbol{\delta}^{n}+\frac{1}{2}\left(\boldsymbol{\delta}^{n+1}-\boldsymbol{\delta}^{n}\right), \quad\left(\boldsymbol{\sigma}^{*}\right)^{n+1}=\boldsymbol{\sigma}^{n}+\frac{1}{2}\left(\boldsymbol{\sigma}^{n+1}-\boldsymbol{\sigma}^{n}\right),
$$

where

$$
\boldsymbol{\delta}^{n}=\left(\delta_{-1}^{n}, \delta_{0}^{n}, \ldots, \delta_{N}^{n}\right)^{T}, \quad \boldsymbol{\sigma}^{n}=\left(\sigma_{-1}^{n}, \sigma_{0}^{n}, \ldots, \sigma_{N}^{n}\right)^{T}
$$


2.2. The cubic B-spline collocation method (CBCM). The Burgers' equation is splitted for the time variable into

$$
\begin{aligned}
& U_{t}+2 U U_{x}=0, \\
& U_{t}-2 \nu U_{x x}=0 .
\end{aligned}
$$

The time-splitted Burgers' equation includes the second-order derivatives. So we should select the cubic B-splines for the trial functions in the collocation method. This selection provides the continuity of up to second order of the trial functions.

The forms of the cubic B-splines $Q_{m}, m=-1, \ldots, N+1$, are defined over the interval $[a, b]$ as follows:

$$
Q_{m}(x)=\frac{1}{h^{3}} \begin{cases}\left(x-x_{m-2}\right)^{3}, & {\left[x_{m-2}, x_{m-1}\right],} \\ h^{3}+3 h^{2}\left(x-x_{m-1}\right)+3 h\left(x-x_{m-1}\right)^{2}-3\left(x-x_{m-1}\right)^{3}, & {\left[x_{m-1}, x_{m}\right],} \\ h^{3}+3 h^{2}\left(x_{m+1}-x\right)+3 h\left(x_{m+1}-x\right)^{2}-3\left(x_{m+1}-x\right)^{3}, & {\left[x_{m}, x_{m+1}\right],} \\ \left(x_{m+2}-x\right)^{3}, & {\left[x_{m+1}, x_{m+2}\right],} \\ 0, & \text { otherwise. }\end{cases}
$$

We seek an approximating solution of the time-splitted Burgers' equation of this form:

$$
U_{N}(x, t)=\sum_{m=-1}^{N+1} \delta_{m}(t) Q_{m}(x) .
$$

The coefficients $\delta_{m}$ are found by requiring that $U_{N}$ satisfies (2.17) at $N+1$ collocation points and boundary conditions.

Nodal value $U$, the first derivative $U^{\prime}$, and the second derivative $U^{\prime \prime}$ at the knots $x_{m}$ are obtained using the expression (2.19) and cubic B-splines $Q_{m}(x)(2.18)$ in terms of the element parameters by

$$
\begin{gathered}
U_{m}=\delta_{m-1}+4 \delta_{m}+\delta_{m+1}, \\
h U_{m}^{\prime}=3\left(\delta_{m+1}-\delta_{m-1}\right), \\
h^{2} U_{m}^{\prime \prime}=6\left(\delta_{m-1}-2 \delta_{m}+\delta_{m+1}\right),
\end{gathered}
$$

where ', " denote the first and the second differentiations with respect to $x$, respectively.

To apply the collocation method, collocation points are selected to coincide with knots and then substituting nodal values $U_{m}$ and first two successive derivatives $U_{m}^{\prime}, U_{m}^{\prime \prime}$ into (2.17). This yields the following coupled matrix system of the first-order ordinary differential equations:

$$
\begin{gathered}
\stackrel{\circ}{\delta}_{m-1}+4 \stackrel{\circ}{\delta}_{m}+\stackrel{\circ}{\delta}_{m+1}+\frac{6}{h} z_{m}\left(-\delta_{m-1}+\delta_{m+1}\right)=0, \\
\stackrel{\circ}{\delta}_{m-1}+4 \stackrel{\circ}{\delta}_{m}+\stackrel{\circ}{\delta}_{m+1}-\frac{12}{h^{2}} \nu\left(\delta_{m-1}-2 \delta_{m}+\delta_{m+1}\right)=0,
\end{gathered}
$$

where $\circ$ denotes derivative with respect to time and $z_{m}=\delta_{m-1}+4 \delta_{m}+\delta_{m+1}$ is the nonlinear term of (2.21). 
Assume that the vector of parameters $\delta_{m}$ and their time derivatives are linearly interpolated between two time levels $n$ and $n+1 / 2$ for $(2.21)$ as

$$
\delta_{m}=\frac{\delta_{m}^{n}+\delta_{m}^{n+1 / 2}}{4}, \quad \delta_{m}^{\circ}=\frac{1}{\Delta t}\left(\delta_{m}^{n+1 / 2}-\delta_{m}^{n}\right),
$$

and parameters $\delta_{m}$ and their time derivatives are interpolated between two time levels $n+1 / 2$ and $n+1$ for $(2.22)$ as

$$
\delta_{m}=\frac{\delta_{m}^{n+1}+\delta_{m}^{n+1 / 2}}{4}, \quad \delta_{m}^{\circ}=\frac{\delta_{m}^{n+1}-\delta_{m}^{n+1 / 2}}{\Delta t} .
$$

Plugging expressions (2.23)-(2.24) up into (2.21)-(2.22), respectively, leads to a nonlinear system of equations each having $N+1$ equations in $N+3$ unknown parameters:

$$
\begin{gathered}
\alpha_{1} \delta_{m-1}^{n+1 / 2}+\alpha_{2} \delta_{m}^{n+1 / 2}+\alpha_{3} \delta_{m+1}^{n+1 / 2}=\alpha_{3} \delta_{m-1}^{n}+\alpha_{2} \delta_{m}^{n}+\alpha_{1} \delta_{m+1}^{n} \\
\alpha_{4} \delta_{m-1}^{n+1}+\alpha_{5} \delta_{m}^{n+1}+\alpha_{4} \delta_{m+1}^{n+1}=\alpha_{6} \delta_{m-1}^{n+1 / 2}+\alpha_{7} \delta_{m}^{n+1 / 2}+\alpha_{6} \delta_{m+1}^{n+1 / 2}
\end{gathered}
$$

where

$$
\begin{array}{lll}
\alpha_{1}=4 h-6 d \Delta t, & \alpha_{2}=16 h+24 \mu, & \alpha_{3}=4 h+6 d \Delta t, \\
\alpha_{4}=h^{2}-3 v \Delta t, & \alpha_{5}=4 h^{2}+6 v \Delta t, & \alpha_{6}=h^{2}+3 v \Delta t .
\end{array}
$$

To have solvable system (2.25)-(2.26), application of the boundary conditions $U(a$, $t)=U_{0}, U(b, t)=U_{N}$ helps us to eliminate the parameters $\delta_{-1}^{n+1 / 2}=U_{0}-\delta_{0}^{n+1 / 2}, \delta_{N}^{n+1 / 2}=$ $U_{N}-\delta_{N-1}^{n+1 / 2}$ from (2.25)-(2.26) so that $(N+1) \times(N+1)$ tridiagonal band matrix equation can be solved with the Thomas algorithm. By having found the parameters $\delta_{m}^{n+1 / 2}$, $m=-1, \ldots, N+1$, from the system $(2.25)$, solution parameters $\delta_{m}^{n+1}$ are obtained from the system (2.26).

Before carrying on obtaining solution parameters, we have to find initial parameters $\delta_{m}^{0}$ by using initial and boundary conditions:

$$
\begin{gathered}
\left(U_{x}\right)_{N}\left(x_{0}, 0\right)=\frac{3}{h}\left(\delta_{1}-\delta_{-1}\right)=U_{x}\left(x_{0}, 0\right), \\
U_{N}\left(x_{j}, 0\right)=\delta_{m-1}+4 \delta_{m}+\delta_{m+1}=U\left(x_{j}, 0\right), \quad j=0, \ldots, N, \\
\left(U_{x}\right)_{N}\left(x_{N}, 0\right)=\frac{3}{h}\left(\delta_{N+1}-\delta_{N-1}\right)=U_{x}\left(x_{N}, 0\right) .
\end{gathered}
$$

The above equations yield a tridiagonal band matrix system whose solution can also be found using the Thomas algorithm. Once we find an approximation $\delta_{m}^{0}$ using the systems (2.28), the rest of the time parameters $\delta_{m}^{n}$ is computed from the algebraic systems (2.25)(2.26). Before proceeding to the each next time step $\delta^{n+1}$, the iteration (2.15) should be repeated two or three times for improving the solution of the nonlinear algebraic equation system.

\section{Numerical examples and conclusion}

In this section, we will present numerical results of the Burgers' equation for two test problems. Accuracy of the methods will be measured with discrete $L_{2}$ and $L_{\infty}$ error norms 
by

$$
\left|U-U_{N}\right|_{\infty}=\max _{j}\left|U_{j}-\left(U_{N}^{n}\right)_{j}\right|, \quad\left\|U-U_{N}\right\|_{2}^{2}=h \sum_{j=0}^{N}\left|\left(U_{j}-\left(U_{N}^{n}\right)_{j}\right)^{2}\right| .
$$

(a) Shock-like solution of the Burgers' equation has analytic solution of the form

$$
U(x, t)=\frac{x / t}{1+\sqrt{\left(t / t_{0}\right)} \exp \left(x^{2} / 4 v t\right)}, \quad t \geq 1,0 \leq x \leq 1,
$$

where $t_{0}=\exp (1 / 8 v)$. Initial condition evaluated from (3.2) at time $t=1$ is taken and boundary conditions are used $U(0, t)=U(1, t)=0$. To test effectiveness of the method, the problem is solved with the earlier used data set $h=0.005$ and $\Delta t=0.01, v=0.005$ over the interval $[0,1]$. Program is run up to time $t=3.25$. Results of the QBCM, CBCM and the analytical solution at some times are documented in Table 3.1. Numerical solutions reflect analytical solutions within very acceptable limits. It is seen that results of the QBCM are better than those of the CBCM in terms of the norms. Error norms of earlier times of the results are much better than that of later times. Comparison of the presented results with those referenced in the paper $[1,2]$, when the equation is splitted, shows that there is advantage of using the QBCM due to having less error than results of the cubic B-spline collocation method to the nonsplitted Burgers' equation, but we get the same results with using CBCM. Graphical solutions of both schemes are also given at time $t=3.25$ in Figures 3.1(a)-3.1(b). Absolute error distributions are depicted at time $t=3.25$ in Figure 3.2, from which errors are concentrated at right boundary. This means that selection of the artificial right boundary causes an increase in error.

With the smaller viscosity constant $v=0.0005$, corresponding computational experiments are carried out. Tabulated results are documented in Table 3.2. Once again, QBCM provided less error than the CBCM. Figures 3.3(a)-3.3(b) show us behavior of the numerical solutions at some times for the presented schemes. For the smaller viscosity value, the propagation value is steeper. Those graphs agree with some of the previous studies within plotting accuracy. As the smaller viscosity value is used, errors increase. In Figures 3.4(a)3.4 (b), error distributions of both schemes at time $t=3.25$ are plotted, from which maximum errors are seen at about the center of the shock and measured as about 0.014 for the QBCM and as about 0.021 for $\mathrm{CBCM}$. As for the comparison with the results given in the papers $[1,2]$, a direct application of both the cubic B-spline collocation method and the B-spline Galerkin finite element method to the Burgers' equation provided less error than both of the proposed schemes.

(b) For our second test example, we consider the particular solution of Burgers' equation

$$
U(x, t)=\frac{\alpha+\mu+(\mu-\alpha) \exp \eta}{1+\exp \eta}, \quad 0 \leq x \leq 1, t \geq 0
$$

where

$$
\eta=\frac{\alpha(x-\mu t-\gamma)}{\nu}
$$




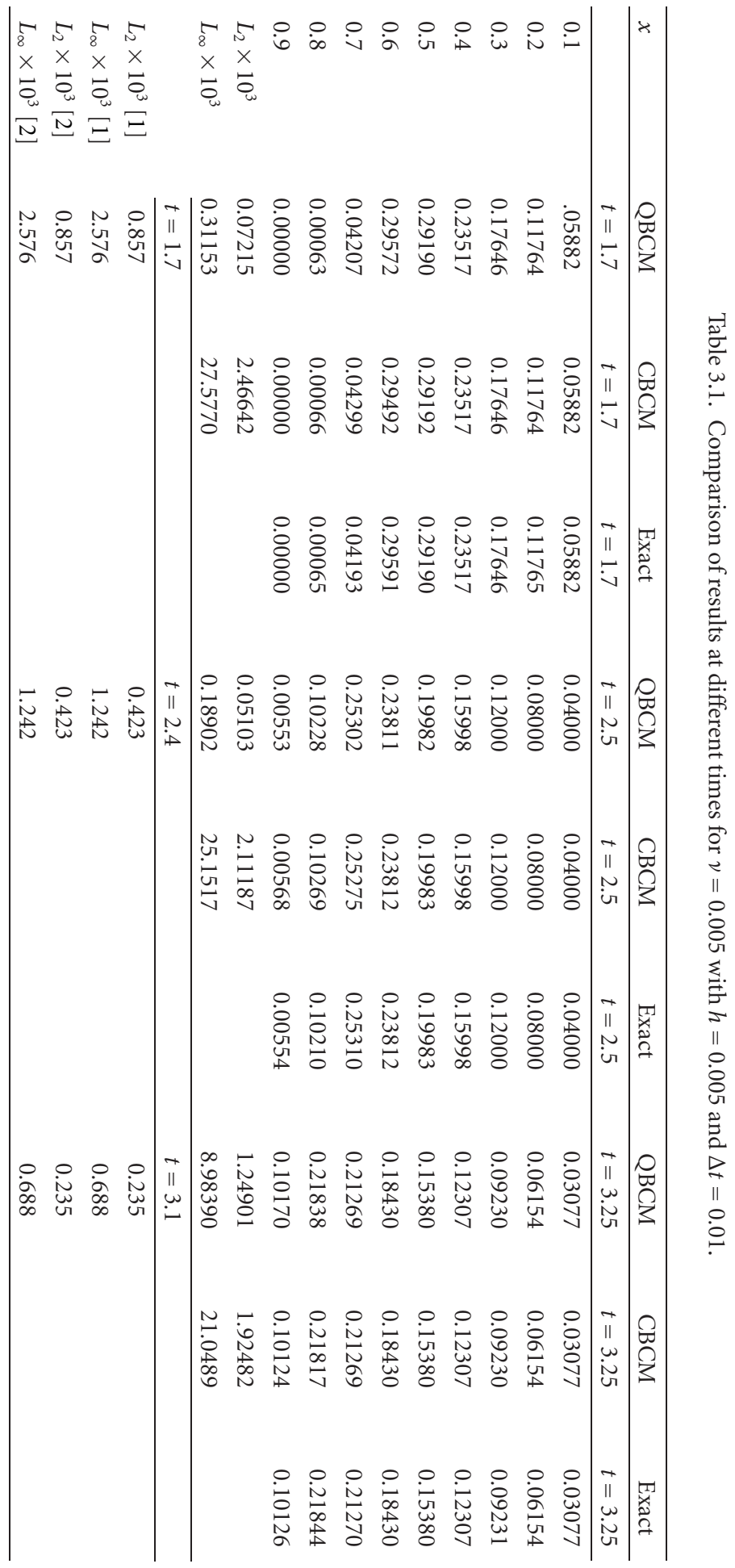


İdris Dağ et al. 529

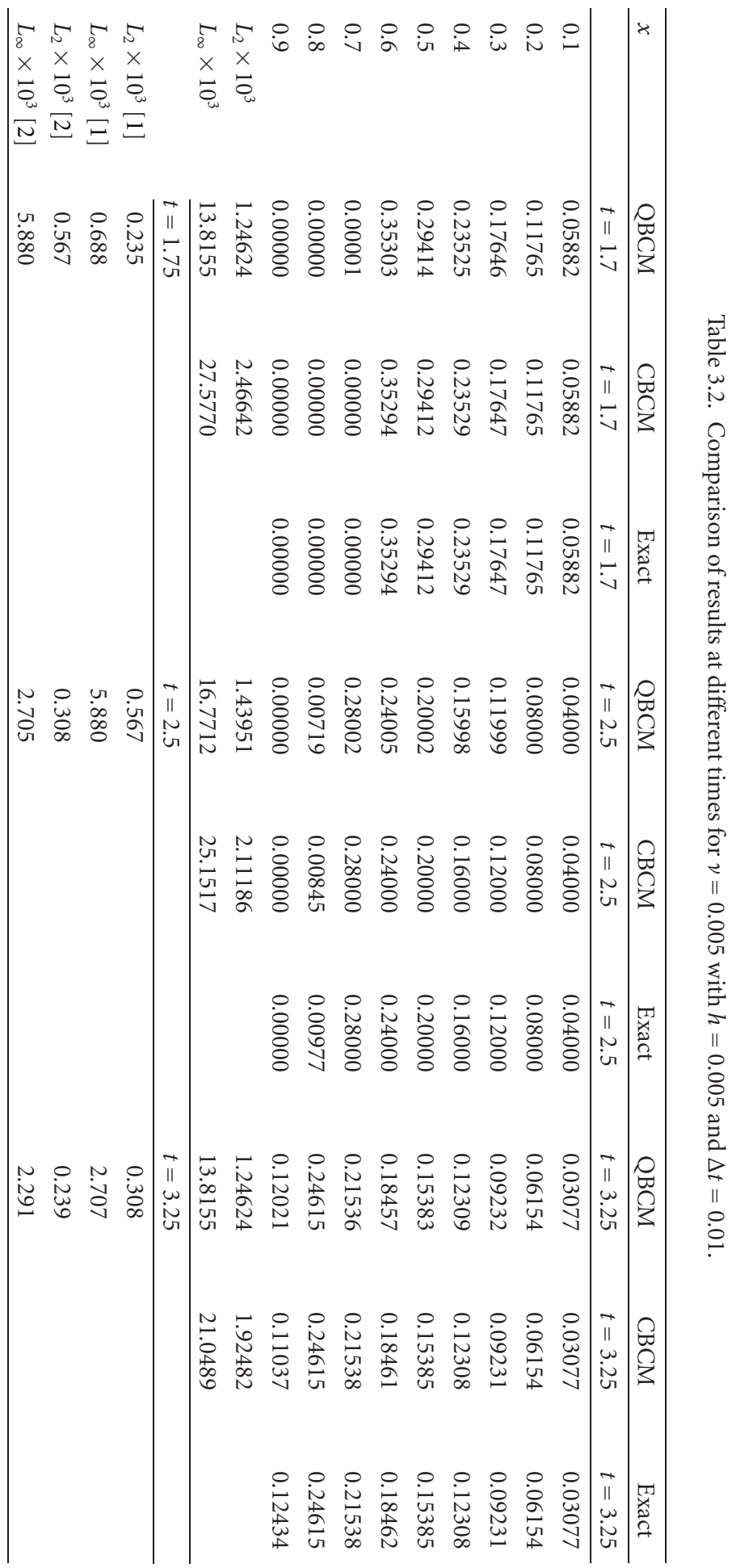




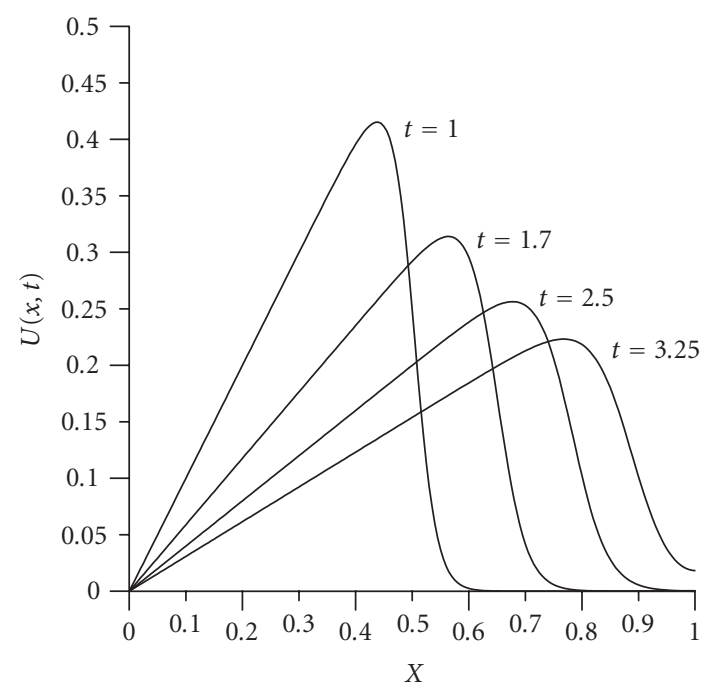

(a) QBCM

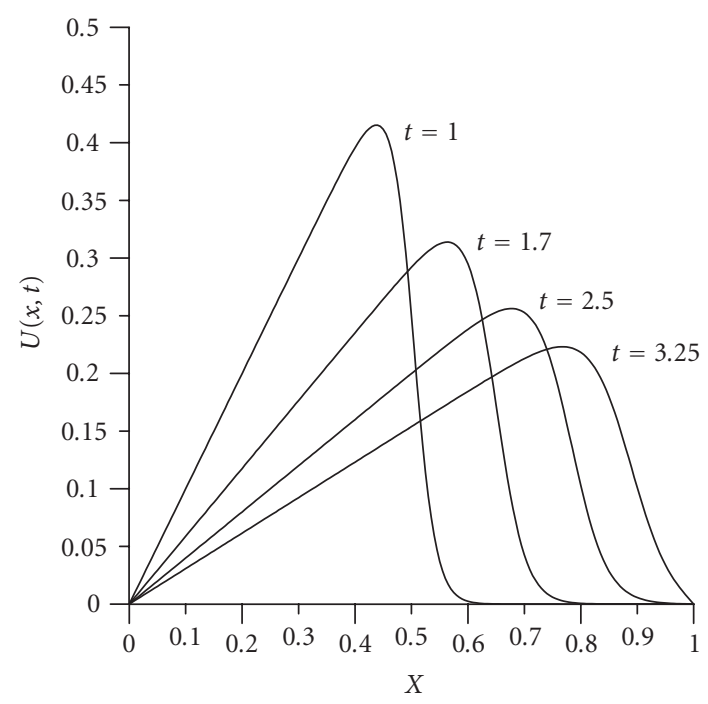

(b) $\mathrm{CBCM}$

Figure 3.1. $v=0.005, h=0.005, \Delta t=0.01$. 
İdris Dağ et al. 531

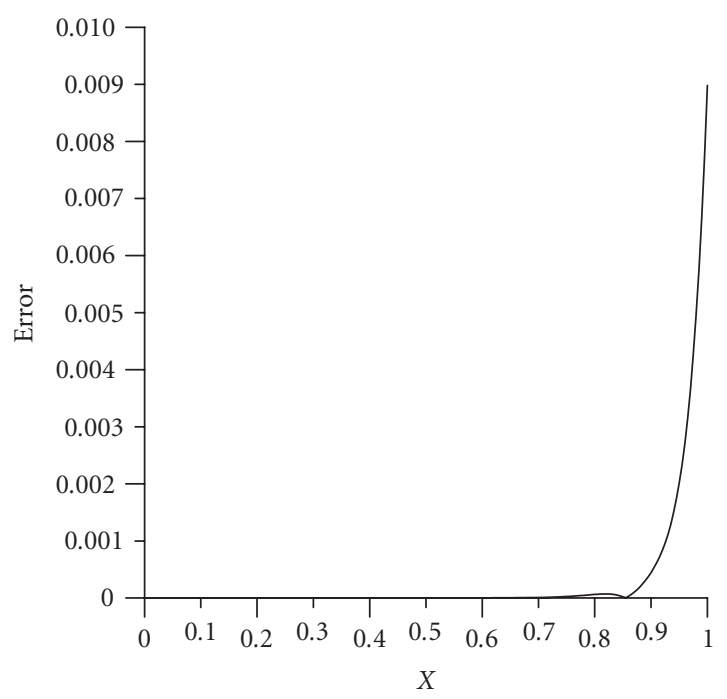

(a) QBCM

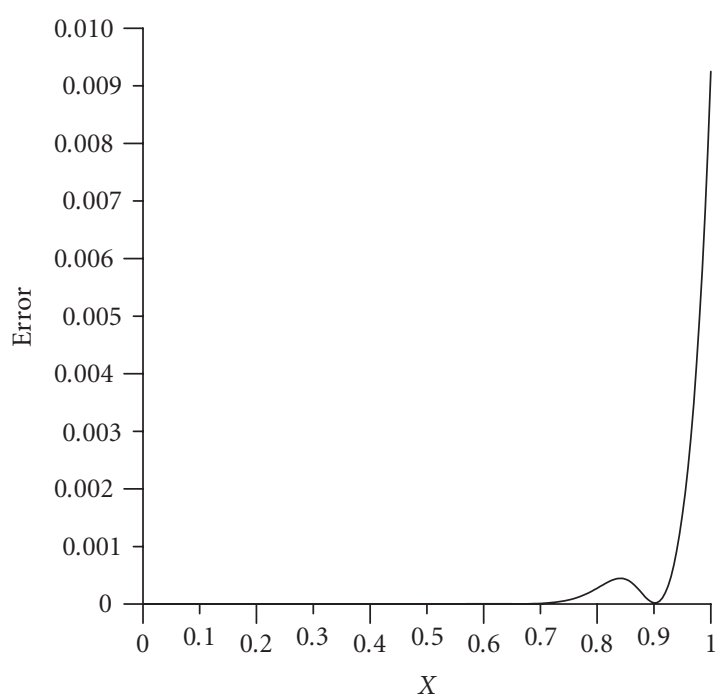

(b) $\mathrm{CBCM}$

Figure 3.2. Errors (|Numerical-Analytical $\mid)$ at time $t=3.25$ with $\nu=0.005$. 
532 B-spline FEM to the Burgers' equation

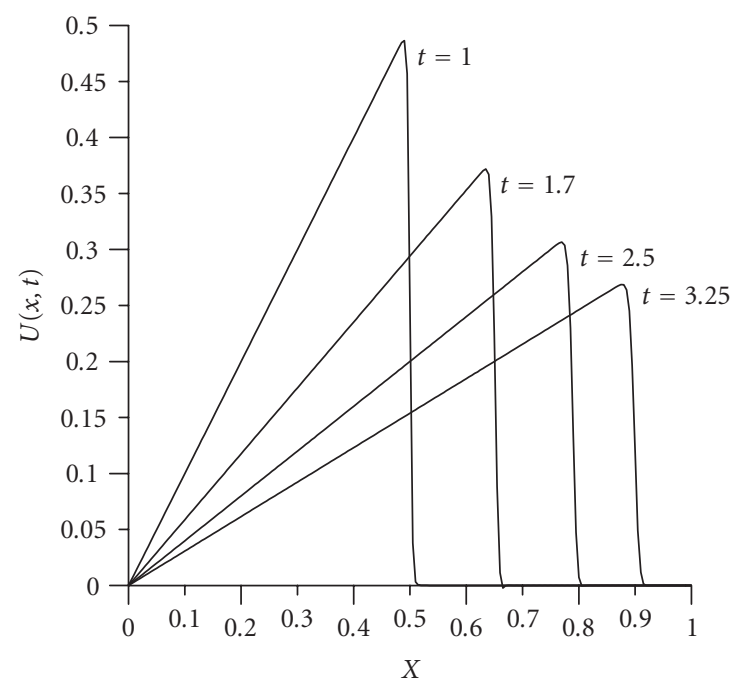

(a) QBCM

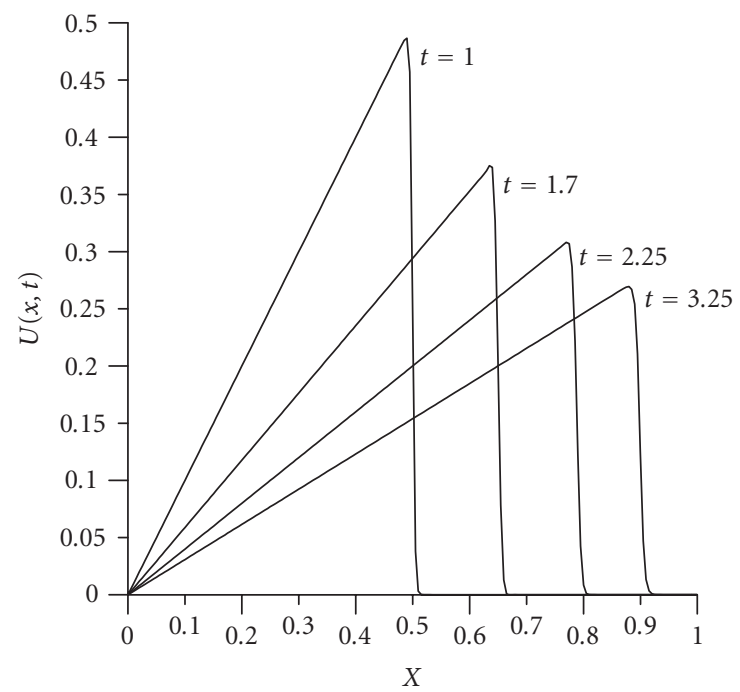

(b) $\mathrm{CBCM}$

Figure 3.3. $v=0.0005, h=0.005, \Delta t=0.01$. 
İdris Dağ et al. 533

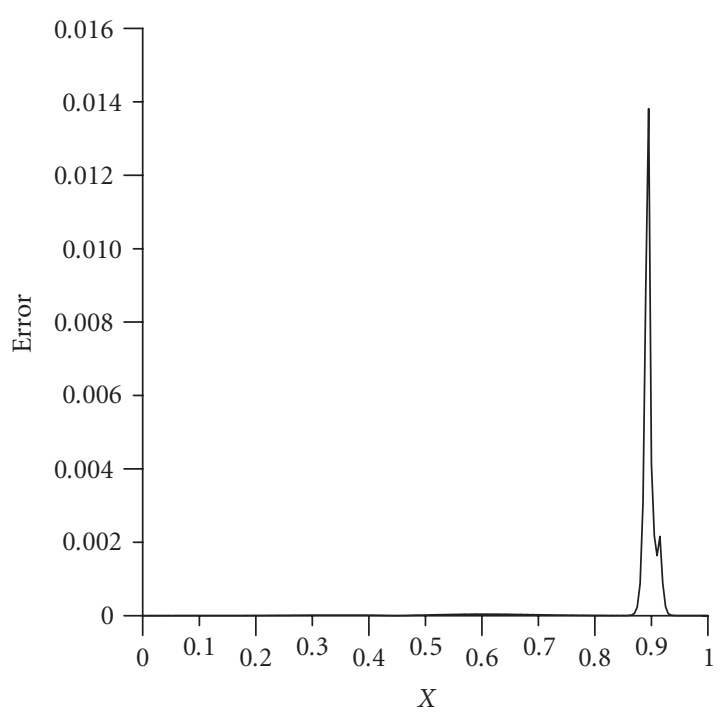

(a) QBCM

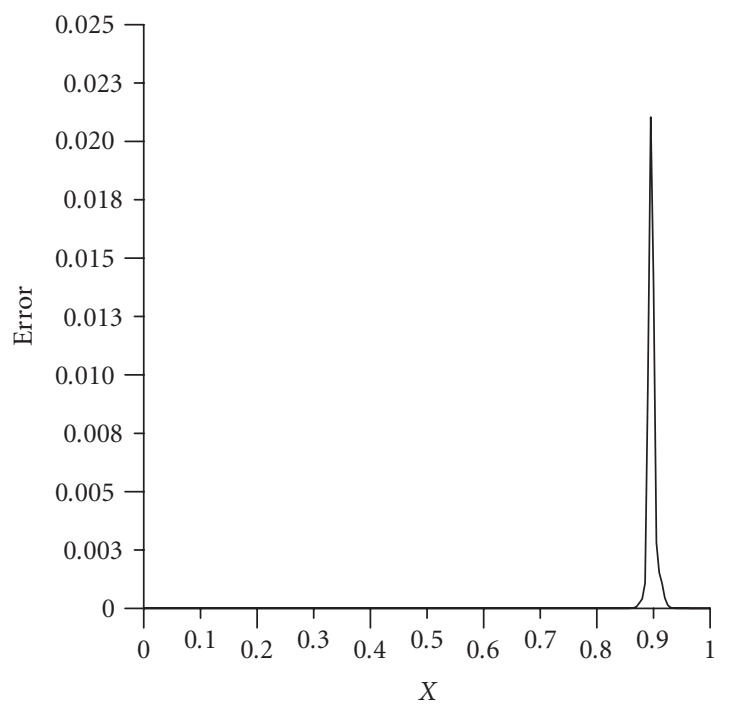

(b) $\mathrm{CBCM}$

Figure 3.4. Errors (|Numerical-Analytical $\mid$ ) at time $t=3.25$ with $v=0.0005$. 
Table 3.3. Comparison of results at time $t=0.5$ for $v=0.01$ with $h=1 / 36$ and $\Delta t=0.01$.

\begin{tabular}{lccc}
\hline$x$ & QBCM & CBCM & Exact \\
\hline 0.000 & 1.000 & 1.000 & 1.000 \\
0.056 & 1.000 & 1.000 & 1.000 \\
0.111 & 1.000 & 1.000 & 1.000 \\
0.167 & 1.000 & 1.000 & 1.000 \\
0.222 & 1.000 & 1.000 & 1.000 \\
0.278 & 0.997 & 1.000 & 0.998 \\
0.333 & 0.977 & 0.983 & 0.980 \\
0.389 & 0.838 & 0.825 & 0.847 \\
0.444 & 0.472 & 0.465 & 0.452 \\
0.500 & 0.237 & 0.244 & 0.238 \\
0.556 & 0.202 & 0.204 & 0.204 \\
0.611 & 0.200 & 0.200 & 0.200 \\
0.667 & 0.200 & 0.200 & 0.200 \\
0.722 & 0.200 & 0.200 & 0.200 \\
0.778 & 0.200 & 0.200 & 0.200 \\
0.833 & 0.200 & 0.200 & 0.200 \\
0.889 & 0.200 & 0.200 & 0.200 \\
0.944 & 0.200 & 0.200 & 0.200 \\
1.000 & 0.200 & 0.200 & 0.200 \\
$L_{2} \times 10^{3}$ & 4.48881 & 5.86664 & \\
$L_{\infty} \times 0^{3}$ & 19.80734 & 22.23450 & \\
\hline & & & \\
\hline
\end{tabular}

and $\alpha, \mu$, and $\gamma$ are constants, which we choose for our experiments $\alpha=0.4, \mu=0.6, \gamma=$ 0.125 . This solution represents a travelling wave and initial condition obtained from the analytic solution moves to the right with speed $\eta$. So the initial condition is determined from (3.3) when $t=0$. The boundary conditions are

$$
U(0, t)=1, \quad U(1, t)=0.2, \quad t \geq 0 .
$$

The simulations are run to time $t=0.5$. Viscosity coefficient $\nu=0.01$, space time $h=$ $1 / 36$, and time step $\Delta t=0.01$ are chosen for computation. Both numerical solutions and analytical solution are given in Table 3.3. It is seen that the agreement between our two numerical solutions and the analytical solution appears satisfactory. Numerical results at various times are graphed in Figures 3.5(a)-3.5(b) for both schemes. The almost same resemblance of wave fronts which was produced from both scheme is noticeable. The QBCM produced slightly better results than the CBCM. Errors of difference between the analytical and numerical results are visualized in Figures 3.6(a)-3.6(b). 
İdris Dağ et al. 535

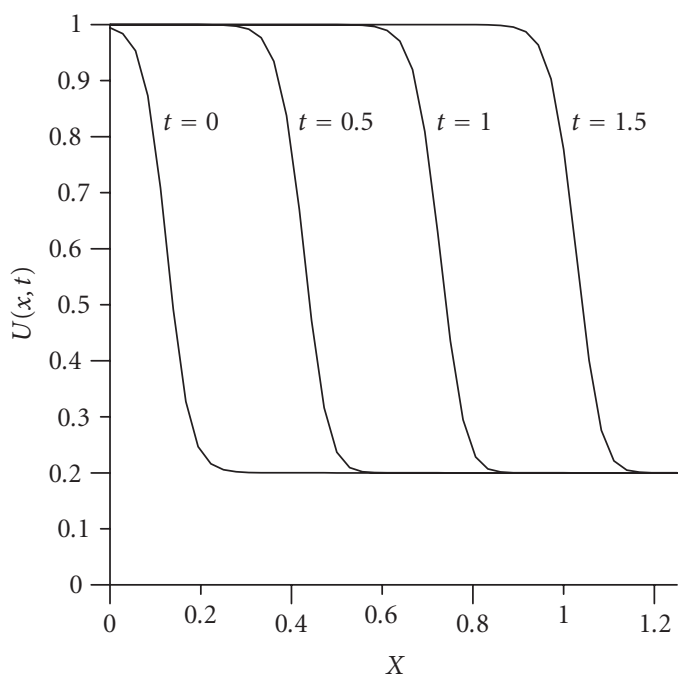

(a) QBCM

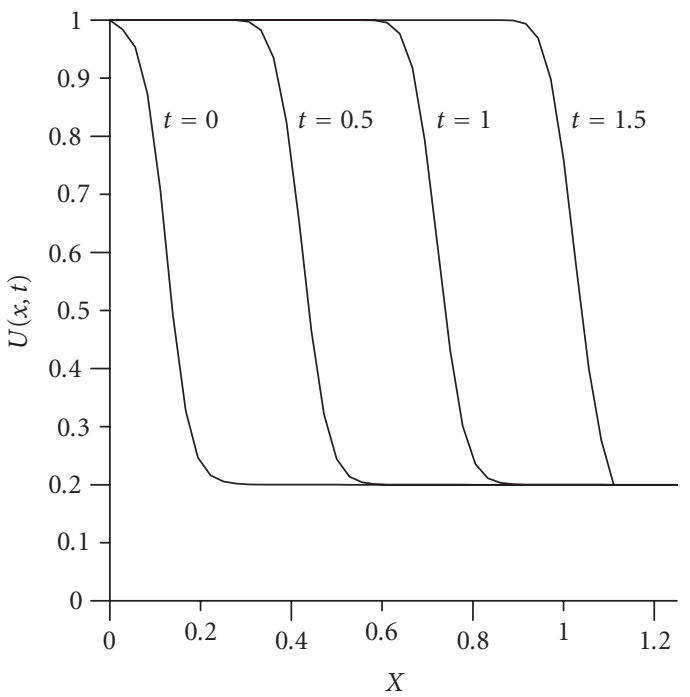

(b) $\mathrm{CBCM}$

Figure 3.5. $v=0.01, h=1 / 36, \Delta t=0.01$. 
536 B-spline FEM to the Burgers' equation

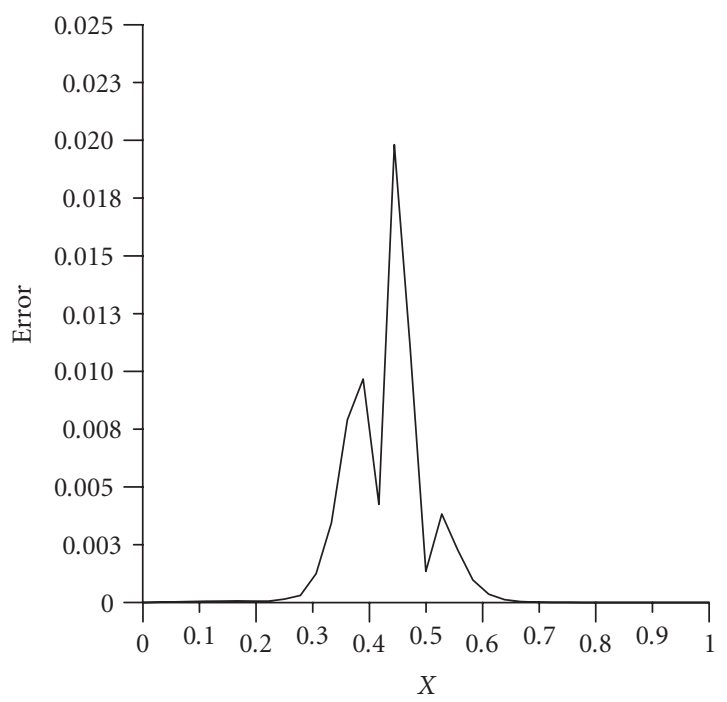

(a) QBCM

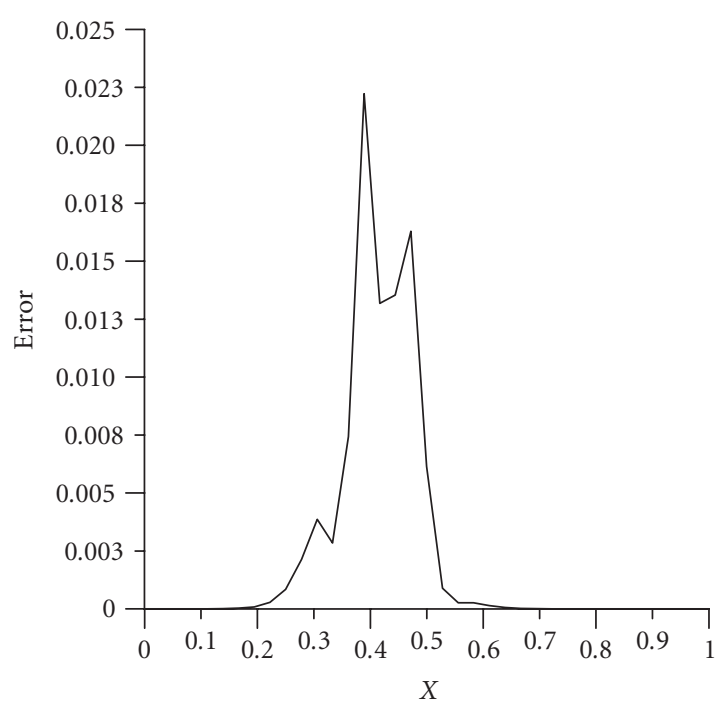

(b) $\mathrm{CBCM}$

Figure 3.6. Errors (|Numerical-Analytical $\mid)$ at time $t=0.5$. 
Numerical solutions of the time-space-splitted Burgers' equation are illustrated when both cubic and quadratic B-spline collocation methods are used. Although both schemes give satisfactory results, the comparison of the two schemes shows that the cubic collocation method for time-splitted Burgers' equation gives less error than the quadratic B-spline collocation method for the space-splitted Burgers' equation. We can also make further comparison of the presented schemes with some of recent previous methods referenced in the paper $[1,2]$. A direct application of those methods to the Burgers' equation produces worse results than the QBCM and almost same results with CBCM. Unfortunaly, the splitting tecnique for the Burgers' equation together with numerical tecnique gives worse results than those of the proposed methods when the smaller values of the viscosity are used. However, when the differential equation involves higher derivatives, time-space-splitted scheme accompanied with low-order polynomial enables to construct some approximate functions in the numerical tecniques so that time-space-splitted numerical methods can be preferable in getting the numerical solution of those differential equations due to providing easy algorithm.

\section{References}

[1] A. H. A. Ali, L. R. T. Gardner, and G. A. Gardner, A Galerkin Approach to the Solution of Burgers' Equation, Maths Preprint Series, no. 90.04, University College of North Wales, Bangor, 1990.

[2] A. H. A. Ali, G. A. Gardner, and L. R. T. Gardner, A collocation solution for Burgers' equation using cubic B-spline finite elements, Comput. Method Appl. M. 100 (1992), no. 3, 325-337.

[3] H. Bateman, Some recent researches on the motion of fluids, Monthly Weather Rec. 43 (1915), no. $4,163-170$.

[4] J. M. Burgers, A mathematical model illustrating the theory of turbulence, Advances in Applied Mechanics, Academic Press, New York, 1948, pp. 171-199.

[5] J. D. Cole, On a quasi-linear parabolic equation occurring in aerodynamics, Q. Appl. Math. 9 (1951), 225-236.

[6] A. M. Davies, Application of the Galerkin method to the solution of the Burgers' equation, Comput. Method Appl. M. 14 (1978), 305-321.

[7] L. R. T. Gardner, G. A. Gardner, and A. H. A. Ali, A method of lines solutions for Burgers' equation, Proceeding of the Asian Pacific Conference on Computational Mechanics, A.A. Balkema/ Rotterdam/ Brookfield, Hong Kong, 1991.

[8] E. Hopf, The partial differential equation $u_{t}+u u_{x}=\mu u_{x x}$, Commun. Pur. Appl. Math. 3 (1950), 201-230.

[9] P. C. Jain and D. N. Holla, Numerical solutions of coupled Burgers' equations, Int. J. Nonlinear Mech. 13 (1978), 213-222.

[10] P. C. Jain and B. L. Lohar, Cubic spline technique for coupled nonlinear parabolic equations, Comput. Math. Appl. 5 (1979), no. 3, 179-185.

[11] P. C. Jain, R. Shankar, and T. V. Singh, Numerical technique for solving convective-reactiondiffusion equation, Math. Comput. Model 22 (1995), no. 9, 113-125.

[12] S. Kutluay, A. Esen, and İ. Dağ, Numerical solutions of the Burgers' equation by the least-squares quadratic B-spline finite element method, J. Comput. Appl. Math. 167 (2004), no. 1, 21-33.

[13] M. J. Lighthill, Viscosity effects in sound waves of finite amplitude, Surveys in Mechanics (G. K. Batchlor and R. M. Davies, eds.), Cambridge University Press, Cambridge, 1956, pp. 250 351 (2 plates).

[14] B. L. Lohar and P. C. Jain, Variable mesh cubic spline technique for $N$-wave solution of Burgers' equation, J. Comput. Phys. 39 (1981), no. 2, 433-442. 


\section{B-spline FEM to the Burgers' equation}

[15] L. A. Pospelov, Propagation of finite-amplitude elastic waves, Soviet Phys. Acoust. 11 (1966), 302-304.

[16] S. G. Rubin and R. A. Graves, Cubic spline approximation for problems in fluid mechanics, Nasa TR R-436, , District of Columbia, 1975.

[17] S. G. Rubin and P. K. Khosla, Higher-order numerical solutions using cubic splines, AIAA J. 14 (1976), no. 7, 851-858.

[18] B. van der Pol, On a non-linear partial differential equation satisfied by the logarithm of the Jacobian theta-functions, with arithmetical applications, Proc. Acad. Sci. Amsterdam 013 (1951), 261-271.

İdris Dağ: Computer Engineering Department, Osmangazi University, 26480 Eskişehir, Turkey E-mail address: idag@ogu.edu.tr

Dursun Irk: Mathematics Department, Osmangazi University, 26480 Eskişehir, Turkey E-mail address:dirk@ogu.edu.tr

Ali Şahin: Mathematics Department, Dumlupınar University, 43100 Kütahya, Turkey E-mail address: asahin@dumlupinar.edu.tr 


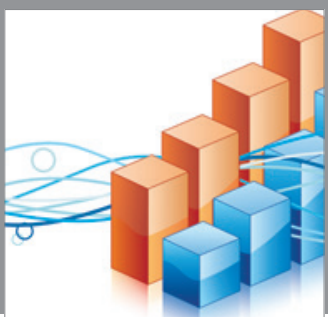

Advances in

Operations Research

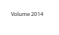

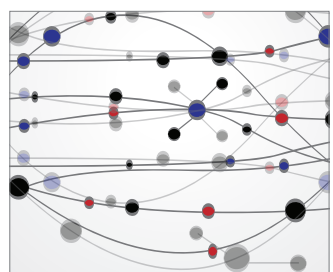

\section{The Scientific} World Journal
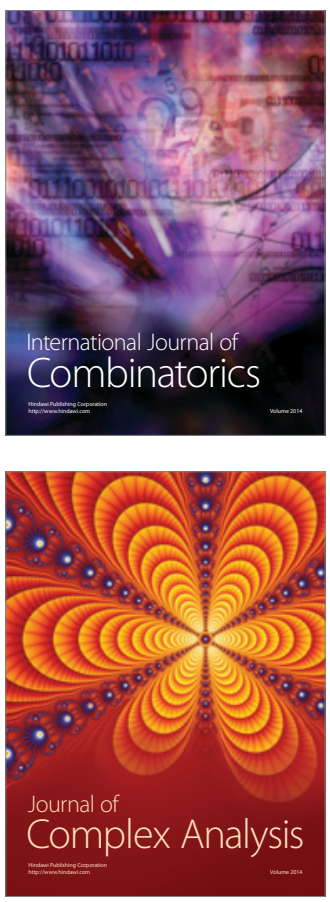

International Journal of

Mathematics and

Mathematical

Sciences
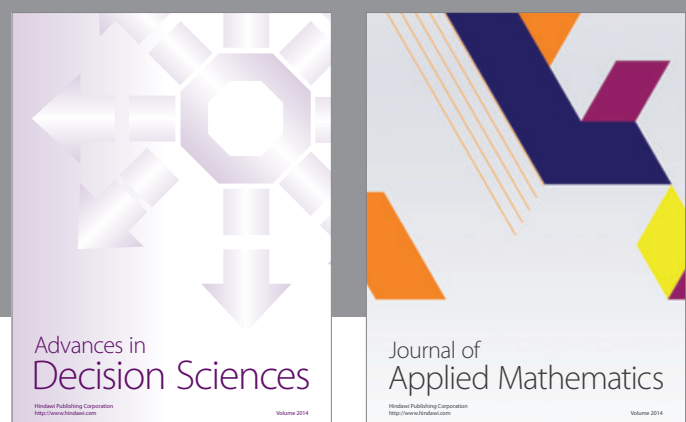

Journal of

Applied Mathematics
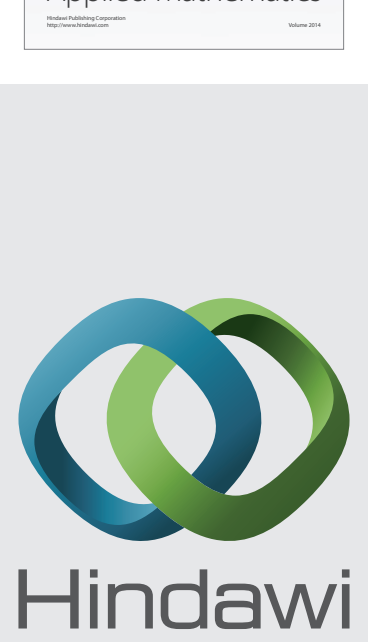

Submit your manuscripts at http://www.hindawi.com
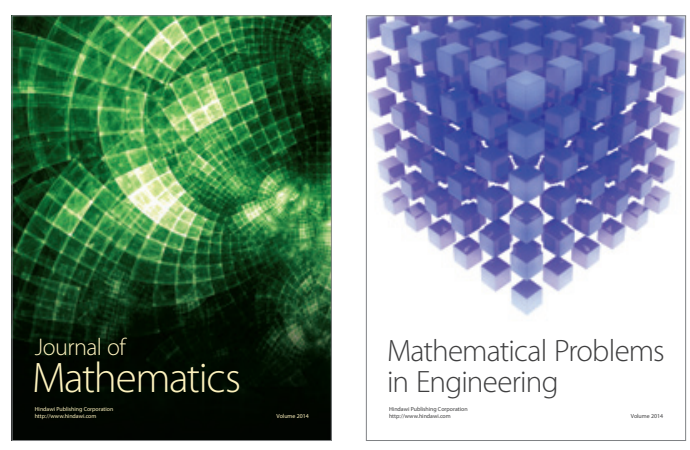

Mathematical Problems in Engineering
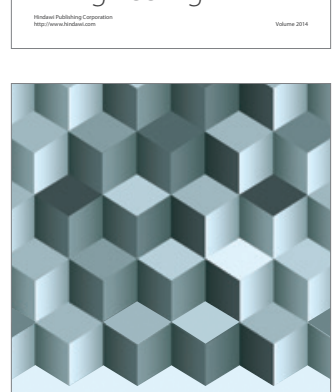

Journal of

Function Spaces
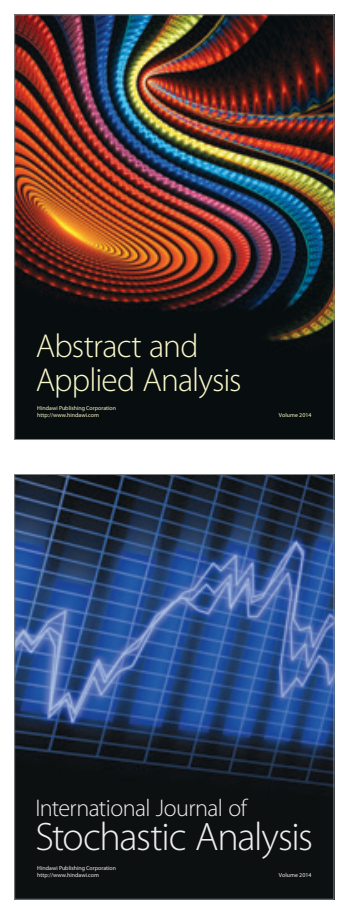

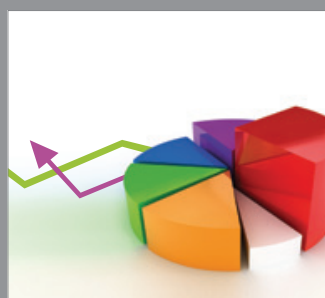

ournal of

Probability and Statistics

Promensencen
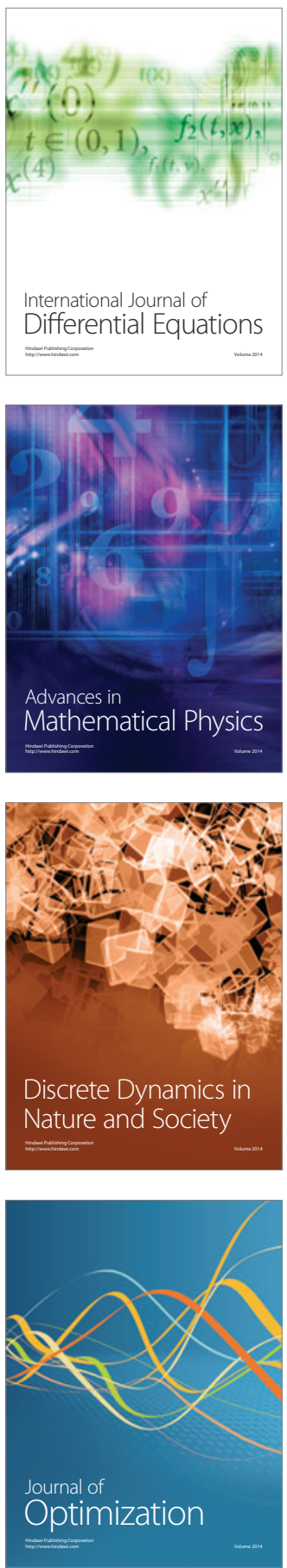